\title{
Safety and efficacy of high-dose versus low-dose aspirin in individuals with ST elevation myocardial infarction undergoing primary percutaneous coronary intervention: A randomized clinical trial
}

\author{
${\text { Abdolhossien Emami Sigaroudi }{ }^{\circledR}, \text { Arsalan Salari }^{\circledR}{ }^{\circledR}, \text { Mohadeseh Poursadeghi }^{1} \text {, Fatemeh Moaddab }}^{2}{ }^{\circledR}$, Seyedeh Fatemeh \\ Mirrazeghi $^{1}$, Fardin Mirbolouk ${ }^{*}{ }^{*}$
}

${ }^{1}$ Cardiovascular Diseases Research Center, Department of Cardiology, Heshmat Hospital, School of Medicine, Guilan University of Medical Sciences, Rasht, Iran

${ }^{2}$ Department of Nursing, Faculty of Nursing and Midwifery, Islamic Azad University, Rasht, Iran

\section{*Correspondence to \\ Fardin Mirbolouk, \\ Email: Mirbolouk@gums.ac.ir, \\ Gums.icrc@gmail.com}

Received 13 Oct. 2019 Accepted 8 Dec. 2019 Published online 26 Dec 2019

Keywords: Aspirin, Patients, Percutaneous coronary intervention, ST elevation myocardial infarction

Abstract
Introduction: Aspirin is the most frequently used antiplatelet therapy after percutaneous coronary intervention
(PCI). Yet, the optimal daily dose of aspirin is unanswered.
Objectives: We aimed to compare the effect of high-dose versus low-dose aspirin in a randomized trial of
patients with ST-segment elevation myocardial infarction (STEMI) undergoing PCI.
Patients and Methods: In a double-blind randomized trial, 175 patients with STEMI were randomly assigned to
high-dose or low-dose aspirin. The primary efficacy outcome was major adverse cardiovascular events (MACE)
as a composite endpoint of death, myocardial infarction, stroke and revascularization procedures. The primary
safety endpoint was major bleeding.
Results: Totally 90 and 85 patients were assigned to high-dose and low-dose aspirin, respectively. The incidence
rate of MACE was 13.1 and 10.1 per 100 person year in high-dose and low dose aspirin, respectively. There was
no significant difference between high-dose and low-dose aspirin in terms of efficacy (Adjusted hazard ratio:
0.85, 95\% CI=0.29-2.45) and safety outcome (Adjusted hazard ratio: $1.65,95 \%$ CI=0.41-6.69).
Conclusion: Efficacy and safety outcomes were not significantly different between high-dose and low-dose
aspirin.
Trial registration: The trial protocol was registered in the Iranian registry of clinical trial (\#IRCT2014122220392N1;
https://en.irct.ir/trial/18085, ethical code; IR.GUMS.REC.1920141901).

Citation: Emami Sigaroudi A, Salari A, Poursadeghi $M$, Moaddab $F$ Mirrazeghi SF, Mirbolouk F. Safety and efficacy of high-dose versus low-dose aspirin in patients with ST elevation myocardial infarction undergoing primary percutaneous coronary intervention: A randomized clinical trial. Immunopathol Persa. 2020;6(1):e05. DOI: 10.15171 ipp. 2020.05

\section{Introduction}

Antiplatelet therapy is the cornerstone of pharmacotherapy after percutaneous coronary intervention (PCI) for long-term success of revascularization procedure (1). Among several drugs, aspirin has been widely accepted in PCI with a history that goes back to 1988 with a significant reduction of the risk of re-infarction $(2,3)$. Nonetheless, the optimal daily dose of aspirin is still unanswered and in practice, the daily maintenance dose is ranged between 75 and $325 \mathrm{mg}$ (4). Most observational studies have shown similar efficacy of high-dose and low dose aspirin. However, in terms of safety, some complications especially increased risks of bleeding from gastrointestinal tract have been reported for higher dose of aspirin (510). Meanwhile, there is scant randomized trial to compare the safety and efficacy of different aspirin doses in patients with ST-

\section{Key point}

In a double-blind randomized trial with one year complete follow-up, results revealed that patients in low-dose aspirin had similar long-term major adverse cardiovascular events compared to high-dose group.

segment elevation myocardial infarction (STEMI) who underwent PCI (11). There is also obvious geographical variation in prescribing dose between countries while most physicians prefer to prescribe higher doses due to concerns of increased risk for subacute ischemic event that outweigh the bleeding complications (4).

\section{Objectives}

In the current study we sought to compare high-dose versus low dose aspirin in a randomized trial of patients with myocardial infarction undergoing primary PCI to

\footnotetext{
Copyright (C) 2020 The Author(s); Published by Nickan Research Institute. This is an open-access article distributed under the terms of the Creative Commons Attribution License (http://creativecommons.org/licenses/by/4.0), which permits unrestricted use, distribution, and reproduction in any medium, provided the original work is properly cited.
} 
provide the best evidence for choosing the suitable dose of aspirin.

\section{Patients and Methods Study population}

This is a double-blind parallel randomized clinical trial designed to compare the long-term safety and efficacy of high versus low-dose aspirin. Study subjects are patients with STEMI who underwent primary PCI. STEMI was defined as typical chest pain lasting for $>30$ min and ST-segment elevation $>1 \mathrm{~mm}$ in $>2$ contiguous electrocardiographic leads. Patients with a history of gastrointestinal bleeding and hemorrhagic stroke, major surgeries within 6 weeks, opium and alcohol abusers, patients with coagulopathies or history of anticoagulant therapy, platelet $<100000 / \mu \mathrm{L}$, hematocrit $<25 \%$, and creatinine $>4 \mathrm{mg} / \mathrm{dL}$ were excluded from the study.

All patients were given a loading dose of $325 \mathrm{mg}$ aspirin orally and $5000 \mathrm{U}$ intravenous bolus of heparin before transportation to the PCI. PCI procedure was performed with a standard femoral approach. One hundred seventyfive eligible patients were then randomly allocated to receive $325 \mathrm{mg}$ daily as high-dose or $81 \mathrm{mg}$ daily as low-dose aspirin for days 2 to 30. Drugs were provided in similar boxes labeled as A or B. Both patients and researchers who assessed the outcome events were blinded about the type of enclosed drugs. At discharge, patients were prescribed for clopidogrel $75 \mathrm{mg} / \mathrm{d}$ for one month or one year, which depended on the type of stent. A checklist including baseline variables, conventional cardiovascular risk factors and laboratory test results were collected for all patients. Transthoracic echocardiography was performed in all patients within 48 hours after PCI and left ventricular ejection fraction was measured based on modified Simpson's method (12).

Patients were assessed daily until the date of discharge from the hospital and then followed-up through telephone contacts. The research staff was blinded to the treatment groups. The primary efficacy endpoint was defined as a composite measure of major adverse cardiovascular events (MACE). The outcomes included in MACE were allcause mortality, revascularization procedures, myocardial infarction (MI), and stroke. All-cause mortality defined as any post-procedural death and was considered of cardiac origin unless there was documentation for another cause. Revascularization procedures defined as subsequent percutaneous intervention or surgery after PCI. Stroke was defined as any ischemic neurologic deficit lasting more than 24 hours. MI defined as prolonged chest pain. The primary safety endpoint was defined as major bleeding event including gastrointestinal (GI) bleeding and bleeding not related to GI system.

\section{Ethical issues}

The research followed the tenets of the Declaration of Helsinki. This paper was extracted from the residential thesis of Mohadeseh Poursadeghi, Department of Cardiology, Heshmat Hospital, School of Medicine, Guilan University of Medical Sciences. Moreover, the study protocol was registered in the Iranian Registry of Clinical Trials (\#IRCT2014122220392N1; https://en.irct. ir/trial/18085.The study was approved by the ethics committee of the Guilan University of Medical Sciences (\#IR.GUMS.REC.1920141901). Accordingly, informed consent was obtained from all the patients.

\section{Statistical analysis}

The analyses were based on intention to treat approach in which all patients are analyzed according to randomization assignment. Baseline variables were compared using t-test or $\chi^{2}$ test. The outcome of interest is the time to endpoint events. The cumulative hazards for each group were estimated using Kaplan-Meier method and were compared using log-rank test. Cox proportional hazard model was used to investigate hazard ratio with $95 \%$ confidence interval of aspirin dosing groups adjusted for potential confounders. For multivariate adjustment, those variables with $P$ value less than 0.1 in univariate analysis were included in the model. Proportional hazard assumption was assessed using graphical approach and goodness of fit test. The global goodness of fit of the model was assessed using likelihood ratio test. All statistical analyses were performed using Stata/SE version (13).

\section{Results}

Patient enrollment was begun in May 2011. Of 200 patients enrolled in the trial, three patients did not meet inclusion criteria, three patients did not accept to participate and 184 patients were randomly allocated to treatment. These patients were divided into two groups of 92 persons. Of these, 9 were excluded and 175 remained. The remaining patients were divided into two groups of 85 (received $81 \mathrm{mg}$ dose), 90 (received $325 \mathrm{mg}$ dose). Of 9 patients excluded from the study, one person died the first day after PCI. Eight patients did not receive allocated intervention. The flowchart of the patients included in the study is shown in Figure 1.

The mean age of the patients was 57.8 years $(\mathrm{SD}=12.4)$ with predominant percentage of male patients (74\%). Baseline characteristic of patients is illustrated in Table 1. The prevalence of conventional risk factors was similar in the two groups. One hundred thirteen patients received stent, as either Bare-metal stent $(\mathrm{n}=72)$ or drug-eluting stent $(n=41)$. Only two patients received elective PCI. The time from randomization to PCI was the only variable with significant difference between the two groups. Though, the mean time from randomization to PCI in both groups was less than standard time of 90 minutes. Left anterior descending (LAD) followed by ramus was the most frequent vessels treated in both groups. The mean number of vessels treated in low-dose aspirin was significantly lower than high-dose aspirin (Table 1). 


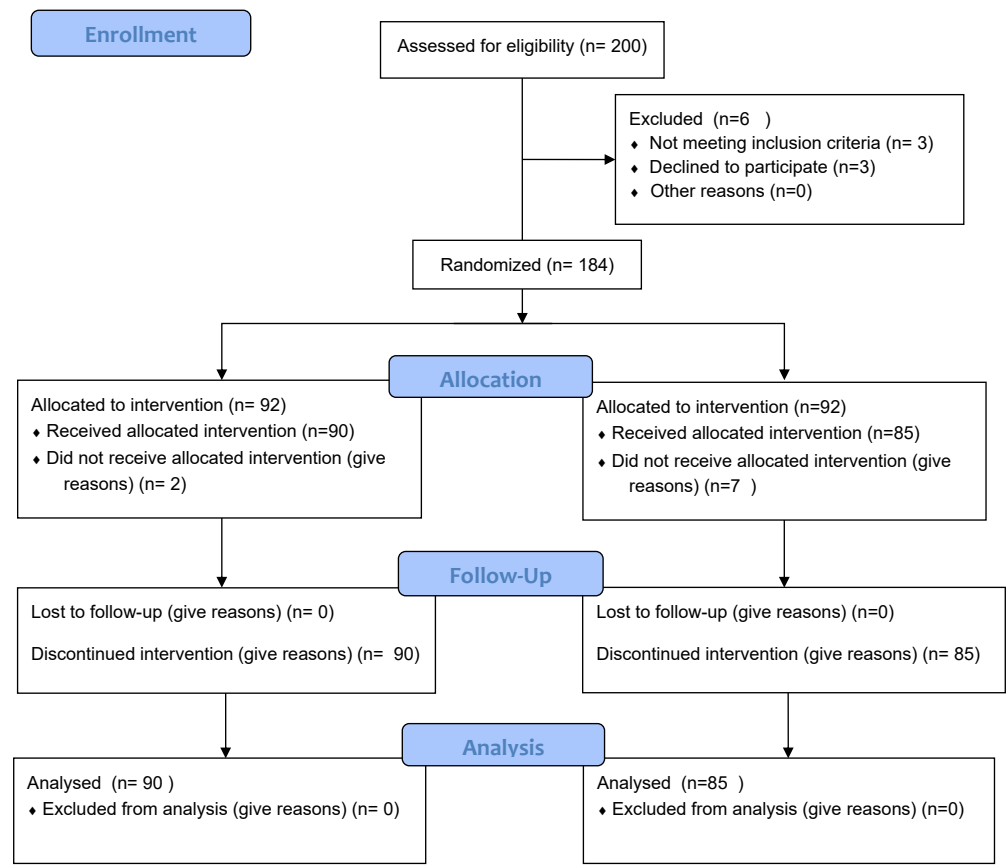

Figure 1. Flow diagram of the study.

Table 1. Baseline characteristic of patients randomized to high and low dose aspirin

\begin{tabular}{|c|c|c|c|}
\hline & $\begin{array}{l}\text { High-dose aspirin } \\
(\mathrm{n}=90)\end{array}$ & $\begin{array}{l}\text { Low-dose aspirin } \\
(n=85)\end{array}$ & $P$ value \\
\hline Age $(y)$ & $59.6(1.29)$ & $55.9(1.35)$ & 0.05 \\
\hline Male gender & $64(71)$ & $65(76)$ & 0.42 \\
\hline Current smoking & $30(33)$ & $40(47)$ & 0.06 \\
\hline Pack-year & $23(3)$ & $25(1.8)$ & 0.64 \\
\hline Hypertension & $36(40)$ & $31(36)$ & 0.63 \\
\hline Diabetes mellitus & $20(22)$ & $25(29)$ & 0.28 \\
\hline Dyslipidemia & $32(35)$ & $34(40)$ & 0.54 \\
\hline $\mathrm{BMI}\left(\mathrm{kg} / \mathrm{m}^{2}\right)$ & $27.1(0.61)$ & $26.9(0.58)$ & 0.81 \\
\hline History of MI & $4(4)$ & $3(3)$ & 0.75 \\
\hline History of stroke & $2(2)$ & $3(3.5)$ & 0.60 \\
\hline Ejection fraction $<30$ & $4(5.5)$ & $5(6.7)$ & 0.76 \\
\hline Troponin & $51(69)$ & $41(67)$ & 0.83 \\
\hline \multicolumn{4}{|l|}{ Stent type } \\
\hline DES & 22 (39) & $19(34)$ & \multirow{2}{*}{0.61} \\
\hline BMS & $35(61)$ & $37(66)$ & \\
\hline No. of vessel treated & $1.97(0.11)$ & $1.62(0.1)$ & 0.02 \\
\hline \multicolumn{4}{|l|}{ Vessels treated } \\
\hline Left main & $11(13)$ & $4(4.9)$ & 0.15 \\
\hline LAD & $60(67)$ & $52(61)$ & 0.45 \\
\hline LCX & $37(43)$ & $24(29)$ & 0.17 \\
\hline RCA & $42(49)$ & $35(43)$ & 0.36 \\
\hline Ramus & $2(2.3)$ & $2(2.4)$ & 0.92 \\
\hline $\begin{array}{l}\text { Time from randomization } \\
\text { to } \mathrm{PCl}(\mathrm{min})\end{array}$ & $65(3.9)$ & $80(4.65)$ & 0.02 \\
\hline
\end{tabular}

Values are mean (SD) or No. (\%).

$\mathrm{BMI}$, body mass index; $\mathrm{MI}$, myocardial infarction; $\mathrm{PCl}$, percutaneous coronary intervention; LAD, Left anterior descending; LCX, circumflex branch of the left coronary artery; RCA, right coronary artery, Ramus $=\mathrm{A}$ branch or subdivision arising from the division (bifurcation) of a blood or lymphatic vessel or a nerve.
A total of 175 person years were followed during one year, which major cardiovascular events (MACEs) occurred among 18 individuals of them. The median duration of follow-up was one year and the incidence rate of MACE was 13.07 per 100 person year in high-dose and 10.1 per 100 person year in low-dose aspirin $(R R=1.29$, 95\% CI $=0.51-3.28)$. The cumulative failure estimates of MACE during one year follow-up were $12 \%$ in high-dose aspirin compared to $9 \%$ in low-dose aspirin. Regarding safety outcomes, the incidence rate of bleeding was 6.5 and 5.2 per 100 person year in high-dose and low-dose aspirin, respectively $(\mathrm{RR}=1.26,95 \%=0.34-4.69)$. There was no significant difference in cumulative failure rate between the two groups (Log rank $P$ value $=0.77$ ). Table 2 shows the frequency of efficacy and safety outcomes in the two groups. Most of the bleeding complications were related to GI bleeding. There were only three cases of genitourinary and skin bleeding occurred in high-dose and low-dose aspirin, respectively.

Multivariate adjustment for potential confounders showed no significant difference in the hazard of MACE between high-dose and low-dose aspirin (adjusted HR). The hazard of bleeding in high-dose group was not significantly different from low-dose aspirin (Table 2).

\section{Discussion}

The finding of current double-blind randomized trial with one year complete follow-up revealed that patients in low-dose aspirin had similar long-term MACE compared to the high-dose group. This finding is in accordance with most of the previous observational studies and a randomized clinical trial which showed no significant 
Table 2. Efficacy and safety outcome of patients randomized to high-dose and low-dose aspirin

\begin{tabular}{lccc}
\hline & $\begin{array}{c}\text { High dose aspirin } \\
\mathbf{n = 9 0}\end{array}$ & $\begin{array}{c}\text { Low dose aspirin } \\
\mathbf{n = 8 5}\end{array}$ & $\begin{array}{c}\text { Unadjusted HR } \\
\mathbf{9 5} \% \mathbf{C l}\end{array}$ \\
\hline MACE & $10(11.2)$ & $8(9.4)$ & $1.26(0.49-3.19)$ \\
\hline Death & $6(6.7)$ & $3(3.5)$ & $0.85(0.29-2.45)$ \\
\hline MI & 0 & $1(1.2)$ & \\
\hline Stroke & 0 & $1(1.2)$ & \\
\hline Revascularization & & $2(2.3)$ & \\
\multicolumn{1}{c}{ CABG } & $3(3.4)$ & $1(1.2)$ & $1.22(0.33-4.53)$ \\
\hline Stent & $1(1.1)$ & $4(4.7)$ & $1.65(0.41-6.69)$ \\
\hline Major bleeding & $5(5.6)$ & & \\
\hline
\end{tabular}

Values are No. (\%).

$\mathrm{HR}$, hazard ratio; $\mathrm{Cl}$, confidence interval; $\mathrm{MI}$, myocardial infarction; $\mathrm{CABG}$, coronary artery bypass graft surgery.

reduction in cardiac outcome for high-dose compared to low dose aspirin (4-11).

Although aspirin is considered as the most frequent antiplatelet therapy in the setting of PCI for patients with acute coronary syndrome, there are scant randomized trials that compared different doses of aspirin directly. Previous randomized trial studies had the primary objective to compare different regimens of antithrombotic agents and assessed the efficacy and safety of aspirin $(9,11,14$ 17). To our knowledge, this is the first randomized trial with primary goal to compare high-dose versus low-dose aspirin. All patients in this study received clopidogrel.

Regarding safety outcome in the present study, there was no difference in the rate of major bleeding. Previous studies showed inconsistent findings on the rate of bleeding as the major complication. Some observational studies assessing different aspirin doses found increased risk of major bleeding associated with higher dose of aspirin $(6,7,18)$. There is also inconsistent finding on the rate of major bleeding as the safety outcome in two previous randomized trial studies $(9,11)$. Some of discrepancies between studies regarding bleeding outcome might be explained by different types of bleeding definition. In this study we excluded occurrence of hematoma during surgery or recovery period. Yu et al found a significant difference in major bleeding between high-dose and low-dose aspirin in a non-randomized study (7). They considered access site hematoma as major bleeding while aspirin side effects might not be initiated immediately after consumption. However, our study is too small to detect significant differences if any, in the rate of bleeding outcome between the two groups.

Despite the consistent finding on similar efficacy outcome between high-dose and low-dose aspirin, there are still variations in recommended guidelines for patients with primary PCI at discharge. There are also regional disparities between clinicians in prescribing practice. Concerns of reduced efficacy for low-dose aspirin at the cost of higher bleeding complication for high-dose aspirin are the two major ambiguities for clinicians to prescribe a suitable dosage. Although this randomized trial with primary randomization of patients based on aspirin dosage found no difference in efficacy and safety outcome, we recommend further randomized trials with larger sample size to detect any difference between the groups, with adequate power.

Though, due to low rate of events, it is estimated that over 11000 patients would be required to show a benefit of high-dose over low-dose aspirin with adequate power (5). We also had about $20 \%$ lost to follow-up in each group. However, the distribution of baseline characteristics and conventional cardiovascular risk factors were not significantly different between lost to follow-up and complete follow-up patients.

\section{Conclusion}

Efficacy and safety outcomes were not significantly different between high-dose and low-dose aspirin.

\section{Study limitations}

The major limitation of current study is small sample size and limited number of available patients used for randomization.

\section{Authors' contribution}

Study concept and design; AES and MP. Acquisition of data; FM and SFM. Statistical analysis; FM. Drafting of the manuscript; SFM and FM. Critical revision of the manuscript for important intellectual content by all authors. All authors read and approved the final version.

Conflicts of interest

There is no conflict of interest in this study.

\section{Ethical considerations}

Ethical issues (including plagiarism, misconduct, data fabrication, falsification, double publication or submission, redundancy) have been completely observed by the authors.

\section{Funding/Support}

This work was supported by Research \& Technology of Guilan University of Medical Sciences in Iran (Grant \# 92040802).

\section{References}

1. Galper BZ, Mauri L. Antiplatelet therapy after coronary stenting. Curr Treat Options Cardiovasc Med. 2013;15(1):1-10. doi: 10.1007/s11936-012-0223-4.

2. Randomised trial of intravenous streptokinase, oral aspirin, both, 
or neither among 17,187 cases of suspected acute myocardial infarction: ISIS-2. ISIS-2 (Second International Study of Infarct Survival) Collaborative Group. Lancet. 1988;2(8607):349360. doi: 10.1016/S0140-6736(88)92833-4

3. Julian DG, Chamberlain DA, Pocock SJ. A comparison of aspirin and anticoagulation following thrombolysis for myocardial infarction (the AFTER study): a multicentre unblinded randomised clinical trial. BMJ (Clinical research ed). 1996;313(7070):1429-31. doi: 10.1136/bmj.313.7070.1429

4. Kohli P, Udell JA, Murphy SA, Cannon CP, Antman EM, Braunwald $E$, et al. Discharge aspirin dose and clinical outcomes in patients with acute coronary syndromes treated with prasugrel versus clopidogrel: an analysis from the TRITON-TIMI 38 study (trial to assess improvement in therapeutic outcomes by optimizing platelet inhibition with prasugrel-thrombolysis in myocardial infarction 38). J Am Coll Cardiol. 2014;63(3):225-32. doi: 10.1016/j.jacc.2013.09.023.

5. So D, Cook EF, Le May M, Glover C, Williams W, Ha A, et al. Association of aspirin dosage to clinical outcomes after percutaneous coronary intervention: observations from the Ottawa Heart Institute PCI Registry. J Invasive Cardiol. 2009;21(3):121-7.

6. Xian Y, Wang TY, McCoy LA, Effron MB, Henry TD, Bach RG, et al. Association of Discharge Aspirin Dose With Outcomes After Acute Myocardial Infarction: Insights From the Treatment with ADP Receptor Inhibitors: Longitudinal Assessment of Treatment Patterns and Events after Acute Coronary Syndrome (TRANSLATE-ACS) Study. Circulation. 2015;132(3):174-81. doi: 10.1161/CIRCULATIONAHA.

7. Yu J, Mehran R, Dangas GD, Claessen BE, Baber U, Xu K, et al. Safety and efficacy of high- versus low-dose aspirin after primary percutaneous coronary intervention in ST-segment elevation myocardial infarction: the HORIZONS-AMI (Harmonizing Outcomes With Revascularization and Stents in Acute Myocardial Infarction) trial. JACC Cardiovasc Interv. 2012;5(12):1231-8. doi: 10.1016/j.jcin.2012.07.016.

8. Harjai KJ, Shenoy C, Orshaw P, Usmani S, Singh M, Boura J, et al. Low-dose versus high-dose aspirin after percutaneous coronary intervention: analysis from the guthrie health offlabel StenT (GHOST) registry. J Interv Cardiol. 2011;24(4):30714. doi: 10.1111/j.1540-8183.2011.00627.x.

9. Jolly SS, Pogue J, Haladyn K, Peters RJ, Fox KA, Avezum A, et al. Effects of aspirin dose on ischaemic events and bleeding after percutaneous coronary intervention: insights from the PCl-CURE study. Eur Heart J. 2009;30(8):900-7. doi: 10.1093/ eurheartj/ehn417.

10. Joyal D, Freihage JH, Cohoon K, Tempelhof M, Leya F, Dieter RS, et al. The influence of low $(81 \mathrm{mg})$ versus high $(325 \mathrm{mg})$ doses of ASA on the incidence of sirolimus-eluting stent thrombosis. J Invasive Cardiol. 2007;19(7):291-4.

11. Mehta SR, Tanguay JF, Eikelboom JW, Jolly SS, Joyner CD, Granger CB, et al. Double-dose versus standard-dose clopidogrel and high-dose versus low-dose aspirin in individuals undergoing percutaneous coronary intervention for acute coronary syndromes (CURRENT-OASIS 7): a randomised factorial trial. Lancet. 2010;376(9748):1233-43. doi: 10.1016/ S0140-6736(10)61088-4.

12. Cheitlin MD, Armstrong WF, Aurigemma GP, Beller GA, Bierman FZ, Davis JL, et al. ACC/AHA/ASE 2003 guideline update for the clinical application of echocardiography: summary article: a report of the American College of Cardiology/American Heart Association Task Force on Practice Guidelines (ACC/AHA/ASE Committee to Update the 1997 Guidelines for the Clinical Application of Echocardiography). Circulation. 2003;108(9):1146-62. doi: 10.1161/01. CIR.0000073597.57414.A9

13. American College of Emergency P, Society for Cardiovascular A, Interventions, O'Gara PT, Kushner FG, Ascheim DD, et al. 2013 ACCF/AHA guideline for the management of ST-elevation myocardial infarction: a report of the American College of Cardiology Foundation/American Heart Association Task Force on Practice Guidelines. Circulation. 2013;61(4):e78-140. doi: 10.1161/CIR.0b013e3182742cf6.

14. Bertrand ME, Legrand V, Boland J, Fleck E, Bonnier J, Emmanuelson $\mathrm{H}$, et al. Randomized multicenter comparison of conventional anticoagulation versus antiplatelet therapy in unplanned and elective coronary stenting. The full anticoagulation versus aspirin and ticlopidine (fantastic) study. Circulation. 1998;98(16):1597-603. doi: 10.1161/01. cir.98.16.1597

15. Bertrand ME, Rupprecht HJ, Urban P, Gershlick AH. Doubleblind study of the safety of clopidogrel with and without a loading dose in combination with aspirin compared with ticlopidine in combination with aspirin after coronary stenting : the clopidogrel aspirin stent international cooperative study (CLASSICS). Circulation. 2000;102(6):624-9. doi: 10.1161/01. cir.102.6.624.

16. Leon MB, Baim DS, Popma JJ, Gordon PC, Cutlip DE, Ho KK, etal. A clinical trial comparing three antithrombotic-drug regimens after coronary-artery stenting. Stent Anticoagulation Restenosis Study Investigators. N Engl J Med. 1998;339(23):1665-71. doi: 10.1056/NEJM199812033392303

17. Schuhlen H, Kastrati A, Pache J, Dirschinger J, Schomig A. Sustained benefit over four years from an initial combined antiplatelet regimen after coronary stent placement in the ISAR trial. Intracoronary Stenting and Antithrombotic Regimen. Am J Cardiol. 2001;87(4):397-400. doi: 10.1016/s00029149(00)01390-4

18. Peters RJ, Mehta SR, Fox KA ,Zhao F, Lewis BS, Kopecky SL, et al. Effects of aspirin dose when used alone or in combination with clopidogrel in patients with acute coronary syndromes: observations from the Clopidogrel in Unstable angina to prevent Recurrent Events (CURE) study. Circulation. 2003; 108(14):1682-7. doi: 10.1161/01.CIR.0000091201.39590.CB 